\title{
Direct detection of odd-frequency superconductivity via time- and angle-resolved photoelectron fluctuation spectroscopy
}

\author{
Viktoriia Kornich $\odot,{ }^{1}$ Frank Schlawin, ${ }^{2,3}$ Michael A. Sentef $\odot,{ }^{2}$ and Björn Trauzettel ${ }^{1}$ \\ ${ }^{1}$ Institut für Theoretische Physik and Astrophysik, Universität Würzburg, 97074 Würzburg, Germany \\ ${ }^{2}$ Max Planck Institute for the Structure and Dynamics of Matter, Center for Free Electron Laser Science (CFEL), Luruper Chaussee 149, \\ 22761 Hamburg, Germany \\ ${ }^{3}$ The Hamburg Centre for Ultrafast Imaging, Luruper Chaussee 149, 22761 Hamburg, Germany
}

(Received 17 June 2021; revised 29 October 2021; accepted 2 November 2021; published 3 December 2021)

\begin{abstract}
We propose a measurement scheme to directly detect odd-frequency superconductivity via time- and angleresolved photoelectron fluctuation spectroscopy. The scheme includes two consecutive nonoverlapping probe pulses applied to a superconducting sample. The photoemitted electrons are collected in a momentum-resolved fashion. Correlations between signals with opposite momenta are analyzed. Remarkably, these correlations are directly proportional to the absolute square of the time-ordered anomalous Green's function of the superconductor. This setup allows for the direct detection of the "hidden order parameter" of odd-frequency pairing. We illustrate this general scheme by analyzing the signal for the prototypical case of a two-band superconductor.
\end{abstract}

DOI: 10.1103/PhysRevResearch.3.L042034

Introduction. Odd-frequency superconductivity is a genuinely dynamic state of matter. It is based on a pairing mechanism in which the two electrons that form a Cooper pair in the superconducting condensate have to correlate with each other at unequal times. This is in contrast to the even-frequency case when the superconducting pairing can be nonvanishing at coinciding times. The most well-known example of even-frequency superconductors are conventional superconductors with $s$-wave spin singlet pairing. Oddfrequency pairing was originally proposed by Berezinskii in the context of helium-3 as a novel type of spin triplet pairing [1]. Subsequently, it was realized by Balatsky and Abrahams that odd-frequency pairing could also exist in a spin singlet version [2], accompanied by other works that transferred the odd-frequency pairing concept to solid-state platforms [3-7]. In the meantime, a number of review articles have emerged that describe odd-frequency superconductivity from different perspectives [8-11]. Moreover, certain experiments have been interpreted as indirect evidence of odd-frequency pairing. Among the well-known examples are the measurement of a long-range supercurrent in Josephson junctions based on $\mathrm{Nb}$, containing the strong ferromagnetic material Co [12], the observation of the polar Kerr effect in the heavy-fermion superconductor $\mathrm{UPt}_{3}$ [13] (in combination with the theoretical analysis performed in Refs. [14,15]), and the measurement of an intrinsic paramagnetic Meissner effect in $\mathrm{Au} / \mathrm{Ho} / \mathrm{Nb}$ trilayer systems [16]. Additionally, it has been argued that signatures of odd-frequency pairing are visible in scanning

Published by the American Physical Society under the terms of the Creative Commons Attribution 4.0 International license. Further distribution of this work must maintain attribution to the author(s) and the published article's title, journal citation, and DOI. tunneling spectra taken on top of magnetic impurities immersed in a $\mathrm{Pb} / \mathrm{Si}(111)$ monolayer [17]. Recent theoretical proposals to detect odd-frequency pairing are, for instance, based on measurements of a supercurrent running from a Majorana scanning tunneling microscope tip to a superconducting substrate [18], transport properties through a quantum spin Hall system in proximity to a $s$-wave superconductor [19], the Josephson current on the surface of Weyl nodal loop semimetals [20], or the Josephson junction current noise [21].

All these examples have in common (with the possible exception of Ref. [21]) that they can only be regarded as an indirect evidence for odd-frequency pairing for the following reason. The defining property of odd-frequency pairing is the mathematical (anti-)symmetry of the time-ordered anomalous Green's function (GF) $F_{\sigma, \sigma^{\prime}}^{\boldsymbol{p}, \boldsymbol{k}}\left(t_{1}, t_{2}\right) \equiv\left\langle T c_{\boldsymbol{p}, \sigma}\left(t_{1}\right) c_{\boldsymbol{k}, \sigma^{\prime}}\left(t_{2}\right)\right\rangle$ (for fermionic annihilation operators with momenta $\boldsymbol{p}, \boldsymbol{k}$ and spins $\sigma, \sigma^{\prime}$, respectively) under the exchange of the two time arguments $t_{1}$ and $t_{2}$. If $F_{\sigma, \sigma^{\prime}}^{p, k}\left(t_{1}, t_{2}\right)$ has an oddfrequency component, which we name $F_{o, \sigma, \sigma^{\prime}}^{\boldsymbol{p}, \boldsymbol{k}}\left(t_{1}, t_{2}\right)$, then the relation $F_{o, \sigma, \sigma^{\prime}}^{\boldsymbol{p}, \boldsymbol{k}}\left(t_{1}, t_{2}\right)=-F_{o, \sigma, \sigma^{\prime}}^{\boldsymbol{p}, \boldsymbol{k}}\left(t_{2}, t_{1}\right)$ holds. In contrast, for the even-frequency component $F_{e, \sigma, \sigma^{\prime}}^{\boldsymbol{p}, \boldsymbol{k}}\left(t_{1}, t_{2}\right)$, the relation $F_{e, \sigma, \sigma^{\prime}}^{\boldsymbol{p}, \boldsymbol{k}}\left(t_{1}, t_{2}\right)=F_{e, \sigma, \sigma^{\prime}}^{\boldsymbol{p}, \boldsymbol{k}}\left(t_{2}, t_{1}\right)$ holds. The odd component $F_{o, \sigma, \sigma^{\prime}}^{\boldsymbol{p}, \boldsymbol{k}}\left(t_{1}, t_{2}\right)$ can be regarded as the "hidden order parameter" of odd-frequency pairing.

In this Letter, we suggest a feasible way to directly detect the absolute square of the anomalous GF, i.e., $\left|F_{\sigma, \sigma^{\prime}}^{\boldsymbol{p}, \boldsymbol{k}}\left(t_{1}, t_{2}\right)\right|^{2}$. If this anomalous GF had even- and odd-frequency components then an exchange of the two time arguments would result in two different signals, proportional to $\mid F_{e, \sigma, \sigma^{\prime}}^{\boldsymbol{p}, \boldsymbol{k}}\left(t_{1}, t_{2}\right) \pm$ $\left.F_{o, \sigma, \sigma^{\prime}}^{\boldsymbol{p}, \boldsymbol{k}}\left(t_{1}, t_{2}\right)\right|^{2}$. This feature is the mathematical working principle behind our detection scheme, which is physically based on time- and angle-resolved photoelectron fluctuation spectroscopy. 


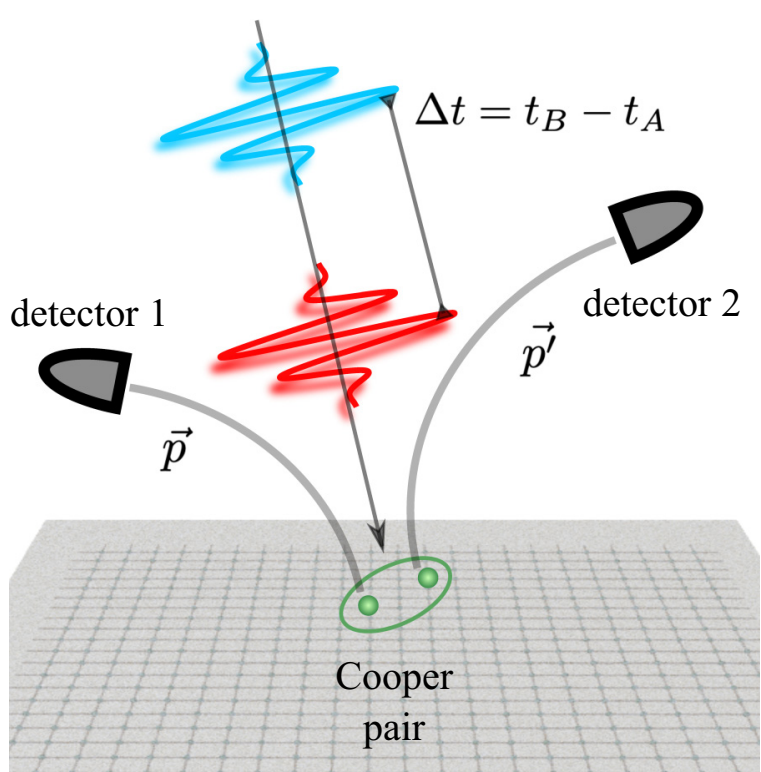

FIG. 1. Schematic of the setup. The superconducting sample is subjected to two separate probe pulses $A$ and $B$ with their temporal envelopes centered at times $t_{A}$ and $t_{B}$, respectively. One possible detection event is the following one: The electron emitted due to pulse $A$ has momentum $\boldsymbol{p}$ and is registered by detector 1 , whereas the electron with momentum $\boldsymbol{p}^{\prime}$ emitted due to the pulse $B$ is registered by detector 2 .

Concretely, we envision a variation of an angle-resolved photoemission spectroscopy (ARPES) setup as shown in Fig. 1. In a static ARPES protocol, a probe photon beam ejects photoelectrons, which are collected in a momentum-resolving detector. This signal is proportional to the expectation value of the photoelectron number with the wave-vector $\boldsymbol{p}$. Here, we instead consider two detectors and two short probe pulses $A$ and $B$. The two detectors can be viewed as being part of a multihit detection scheme, which is able to detect two photoelectrons with momenta $\boldsymbol{p}$ and $\boldsymbol{p}^{\prime}$ within a certain time range [22]. This gives rise to correlation signals of the form $I_{\boldsymbol{p}, \sigma ; \boldsymbol{p}^{\prime}, \sigma^{\prime}}^{(2)}=\left\langle n_{\boldsymbol{p}, \sigma} n_{\boldsymbol{p}^{\prime}, \sigma}\right\rangle$. A similar setup-with a single probe pulse - was analyzed by Stahl and Eckstein [23], who showed that such correlation signals can probe the anomalous GF of superconductors. In the single probe pulse case, however, it is not possible to exchange the time arguments $t_{1}$ and $t_{2}$ of the anomalous GF. As discussed above, this exchange is essential to directly probe odd-frequency pairing and specified below.

Model. The Hamiltonian describing photoemission of electrons due to the two probe pulses $A$ and $B$ can be written as

$$
\begin{aligned}
H= & \sum_{\boldsymbol{k}, \boldsymbol{p}, \sigma, \sigma^{\prime}} S_{A}^{*}(t) e^{i \Omega_{A} t} M_{\boldsymbol{k}, \boldsymbol{p}}^{\sigma, \sigma^{\prime}} a_{\boldsymbol{p}, \sigma^{\prime}}^{\dagger} c_{\boldsymbol{k}, \sigma} \\
& +S_{B}^{*}(t) e^{i \Omega_{B} t} M_{\boldsymbol{k}, \boldsymbol{p}}^{\sigma, \sigma^{\prime}} b_{\boldsymbol{p} \sigma^{\prime}}^{\dagger} c_{\boldsymbol{k}, \sigma}+\text { H.c. }
\end{aligned}
$$

where $c_{k, \sigma}$ is an operator of an electron in the material with momentum $\boldsymbol{k}$ and spin $\sigma$. Likewise, $a_{\boldsymbol{p}, \sigma^{\prime}}$ and $b_{\boldsymbol{p}, \sigma^{\prime}}$ are operators of photoemitted electrons with momenta $\boldsymbol{p}$ and spins $\sigma^{\prime}$ via pulse $A$ and pulse $B$, respectively. The matrix element $M_{\boldsymbol{k}, \boldsymbol{p}}^{\sigma \sigma^{\prime}}$ characterizes emission and is often approximated as $M_{\boldsymbol{k}, \boldsymbol{p}}^{\sigma \sigma^{\prime}}=M_{0} \delta_{\boldsymbol{k}, \boldsymbol{p}} \delta_{\sigma \sigma^{\prime}}$. This approximation is valid if the emitted electrons have the same momentum and spin as they have in the material. The pulses $A$ and $B$ have temporal envelopes $S_{A}(t)$ and $S_{B}(t)$ and frequencies $\Omega_{A}$ and $\Omega_{B}$, respectively. It is important that the pulses do not overlap. The Hamiltonian of emitted electrons via pulse $A$ is

$$
H_{a}=\sum_{\boldsymbol{p}, \sigma} E_{p} a_{\boldsymbol{p}, \sigma}^{\dagger} a_{\boldsymbol{p}, \sigma}=\sum_{\boldsymbol{p}, \sigma} E_{p} n_{\boldsymbol{p}, \sigma}^{a},
$$

and analogously for emitted electrons via pulse $B$. The spectrum of emitted electrons is $E_{p}=p^{2} / 2 m$, where $m$ is the bare mass of an electron.

We consider a material that has superconducting pairing. Hence, the Hamiltonian $H_{c}$ is assumed to contain terms of the kinetic energy of the electron, such as $\xi_{p} c_{\boldsymbol{p}, \sigma}^{\dagger} c_{\boldsymbol{p}, \sigma}$, as well as mean-field terms of the type $\Delta_{\sigma \sigma^{\prime}}^{p} c_{-p, \sigma^{\prime}}^{\dagger} c_{\boldsymbol{p}, \sigma}^{\dagger}$, where $\Delta_{\sigma \sigma^{\prime}}^{p}$ is the superconducting pairing potential. For now, we do not specify $H_{c}$ in more detail because different materials can have different forms of the Hamiltonian that do not affect our general considerations for the detection of odd-frequency pairing significantly.

Photoemission signals. Following the standard description of electron photoemission, we define the total population of the emitted electrons with momentum $\boldsymbol{p}$ and $\operatorname{spin} \sigma$ as

$$
I_{\boldsymbol{p}, \sigma}^{(1)}=\left\langle n_{\boldsymbol{p}, \sigma}\right\rangle_{t=\infty}=\left\langle\mathcal{S}^{\dagger}\left(n_{\boldsymbol{p}, \sigma}^{a}+n_{\boldsymbol{p}, \sigma}^{b}\right) \mathcal{S}\right\rangle_{0},
$$

where the averaging $\langle\cdots\rangle_{0}$ is over the initial state of the system before applying pulses and in the absence of emitted electrons. The evolution matrix is defined as $\mathcal{S}=$ $T \exp \left[-i \int_{-\infty}^{\infty} d \tau H(\tau)\right]$. Since we are interested in the total population, the final time is taken to be $t=\infty$. The statistical correlations of photoemission events from all pulses are

$$
I_{\boldsymbol{p}, \sigma ; \boldsymbol{p}^{\prime}, \sigma^{\prime}}^{(2)}=\left\langle n_{\boldsymbol{p}, \sigma} n_{\boldsymbol{p}^{\prime}, \sigma^{\prime}}\right\rangle_{t=\infty} .
$$

In the case $\boldsymbol{p} \neq \boldsymbol{p}^{\prime}, I_{\boldsymbol{p}, \sigma ; \boldsymbol{p}^{\prime}, \sigma^{\prime}}^{(2)}$ depends on a two-point GF with respect to electrons in the material $G_{\sigma_{2}, \sigma_{1}, \sigma_{2}^{\prime}, \sigma_{1}^{\prime}}^{\boldsymbol{k}_{2}, \boldsymbol{k}_{1}, \boldsymbol{k}_{1}^{\prime}, \boldsymbol{k}_{2}^{\prime}}\left(\tau_{2}, \tau_{1}, \tau_{2}^{\prime}, \tau_{1}^{\prime}\right)=$ $\left\langle\bar{T}\left[c_{\boldsymbol{k}_{2}, \sigma_{2}}^{\dagger}\left(\tau_{2}\right) c_{\boldsymbol{k}_{1}, \sigma_{1}}^{\dagger}\left(\tau_{1}\right)\right] T\left[c_{\boldsymbol{k}_{1}^{\prime}, \sigma_{1}^{\prime}}\left(\tau_{1}^{\prime}\right) c_{\boldsymbol{k}_{2}^{\prime}, \sigma_{2}^{\prime}}\left(\tau_{2}^{\prime}\right)\right]\right\rangle$, where $\bar{T} \mathrm{de}-$ notes antitime ordering, which comes from the expansion of $\mathcal{S}^{\dagger}$ up to second order (see Appendix A for the detailed derivation of the signal). This two-point GF can be simplified via Wick's theorem.

Following the description above, the fluctuations of the correlations are defined as

$$
\Delta I_{\boldsymbol{p}, \sigma ; \boldsymbol{p}^{\prime}, \sigma^{\prime}}=I_{\boldsymbol{p}, \sigma ; \boldsymbol{p}^{\prime}, \sigma^{\prime}}^{(2)}-I_{\boldsymbol{p}, \sigma}^{(1)} I_{\boldsymbol{p}^{\prime}, \sigma^{\prime}}^{(1)} .
$$

In the case of $\boldsymbol{p}^{\prime}=-\boldsymbol{p}$, the particle-number-conserving terms of type $\left\langle c^{\dagger} c\right\rangle_{0}$ cancel, and the signal directly depends on the anomalous GF via

$$
\Delta I_{\boldsymbol{p}, \sigma ;-\boldsymbol{p}, \sigma^{\prime}}=\left|\int_{-\infty}^{\infty} d \tau_{1} d \tau_{2} \Xi\left(\boldsymbol{p}, \tau_{1}, \tau_{2}\right) F_{\sigma^{\prime}, \sigma}^{-\boldsymbol{p}, \boldsymbol{p}}\left(\tau_{1}, \tau_{2}\right)\right|^{2},
$$

where $\Xi\left(\boldsymbol{p}, \tau_{1}, \tau_{2}\right)$ is a function of pulse shapes, frequencies of applied pulses, $M_{0}$, and kinetic energy of emitted electrons (see Appendix A).

We can deduce from Eq. (6) that, applying a single pulse and detecting emitted electrons in the case of $M_{\sigma \sigma^{\prime}}^{\boldsymbol{k}, \boldsymbol{p}}=M_{0} \delta_{\boldsymbol{k}, \boldsymbol{p}} \delta_{\sigma, \sigma^{\prime}}$, this does not enable us to obtain any signal stemming from odd-frequency pairing because in that case $\Xi\left(\boldsymbol{p}, \tau_{1}, \tau_{2}\right)=\Xi\left(\boldsymbol{p}, \tau_{2}, \tau_{1}\right)=M_{0}^{2} S^{*}\left(\tau_{1}\right) S^{*}\left(\tau_{2}\right) e^{i\left(\Omega+E_{p}\right)\left(\tau_{1}+\tau_{2}\right)}$ 
is an even function with respect to an exchange of the two time arguments. Therefore, the convolution with any odd component of the anomalous $\operatorname{GF} F_{\sigma^{\prime}, \sigma}^{-\boldsymbol{p}, \boldsymbol{p}}\left(\tau_{1}, \tau_{2}\right)$ vanishes.

Thus, in order to detect odd-frequency superconductivity, we need to perform a time-resolved measurement, implying $\Xi\left(\boldsymbol{p}, \tau_{1}, \tau_{2}\right) \neq \Xi\left(\boldsymbol{p}, \tau_{2}, \tau_{1}\right)$. To achieve this goal, we suggest to apply two pulses $A$ and $B$ that do not overlap in time. For pedagogical reasons, we first restrict ourselves to a specific process: Detector 1 measures all electrons emitted via pulse $A$ with momentum $\boldsymbol{p}$ and spin $\sigma$, whereas detector 2 measures all electrons emitted via pulse $B$ with momentum $-\boldsymbol{p}$ and spin $\sigma^{\prime}$. In this specific detection scheme, we only need to analyze the term $\Delta I_{\boldsymbol{p}, \sigma ;-\boldsymbol{p}, \sigma^{\prime}}^{a b}=\left\langle n_{\boldsymbol{p}, \sigma}^{a} n_{-\boldsymbol{p}, \sigma^{\prime}}^{b}\right\rangle_{t=\infty}-$ $\left\langle n_{\boldsymbol{p}, \sigma}^{a}\right\rangle_{t=\infty}\left\langle n_{-\boldsymbol{p}, \sigma^{\prime}}^{b}\right\rangle_{t=\infty}$ with

$$
\Xi\left(\boldsymbol{p}, \tau_{1}, \tau_{2}\right)=M_{0}^{2} S_{B}^{*}\left(\tau_{1}\right) S_{A}^{*}\left(\tau_{2}\right) e^{i\left[\Omega_{A} \tau_{2}+\Omega_{B} \tau_{1}+E_{p}\left(\tau_{1}+\tau_{2}\right)\right]} .
$$

As the pulses $A$ and $B$ are separated in time, the inequality $\Xi\left(\boldsymbol{p}, \tau_{1}, \tau_{2}\right) \neq \Xi\left(\boldsymbol{p}, \tau_{2}, \tau_{1}\right)$ holds if the two photons in pulses $A$ and $B$ are distinguishable, for instance, by energy (or any other means).

The temporal envelopes $S_{A}(t)$ and $S_{B}(t)$ are likely to be close to Gaussian shape in real experiments. For simplicity, we assume them to be infinitely short $\delta$ functions, i.e., $S_{A}(t)=S_{0} \delta\left(t-t_{A}\right)$ and $S_{B}(t)=S_{0} \delta\left(t-t_{B}\right)$ [24]. In this case, the signal is

$$
\Delta I_{\boldsymbol{p}, \sigma ;-\boldsymbol{p}, \sigma^{\prime}}^{a b}=\left(S_{0} M_{0}\right)^{4}\left|F_{\sigma^{\prime}, \sigma}^{-\boldsymbol{p}, \boldsymbol{p}}\left(t_{B}, t_{A}\right)\right|^{2} .
$$

Evidently, it can serve as a direct probe to odd-frequency superconductivity: If $F$ has an odd component $F_{o}$ and an even component $F_{e}$ then an exchange of the two time arguments in Eq. (8) allows us to probe $\left|F_{e}+F_{o}\right|^{2}$ and $\left|F_{e}-F_{o}\right|^{2}$.

Depending on the characteristics of the materials and detection schemes, we have to take additional terms into account. For instance, we have to sum over spin degrees of freedom if the detection scheme is not spin-resolved. Moreover, we have to sum over terms, such as $\Delta I^{a a}, \Delta I^{b b}$, and $\Delta I^{b a}$ if they happen to yield a finite signal, and both detectors are able to detect photoelectrons of any of the two types $A$ and $B$. In the example discussed below, the terms $\Delta I^{a a}$ and $\Delta I^{b b}$ do not depend on the time difference and produce just a constant shift of the overall signal, whereas the term $\Delta I^{b a}$ yields an identical signal to $\Delta I^{a b}$.

Two-band superconductor. Below, we demonstrate how our proposal works within a model of a two-band superconductor developed by Parhizgar and Black-Schaffer [25]. It should be kept in mind that our proposal is generally applicable to any odd-frequency superconductor. The demonstration with respect to the two-band superconductor is just an example in which many features can be understood analytically. The number of bands matter for the detection scheme. In the two-band case, the energy difference between the bands can be employed to distinguish the two probe pulses. In the single-band case, this is not an option. Then, other degrees of freedom (e.g., spin) have to be taken into account for this purpose.

The Hamiltonian of a two-band superconductor in the basis $\psi_{\boldsymbol{p}}^{\dagger}=\left(c_{1, \boldsymbol{p}, \uparrow}^{\dagger}, c_{2, \boldsymbol{p}, \uparrow}^{\dagger}, c_{1,-\boldsymbol{p}, \downarrow}, c_{2,-\boldsymbol{p}, \downarrow}\right)^{T}$ can be written as

$$
H_{c}=\left(\xi_{+} \tau_{0}+\xi_{-} \tau_{z}+\xi_{12} \tau_{x}\right) \gamma_{z}+\left(\delta_{+} \tau_{0}+\delta_{-} \tau_{z}\right) \gamma_{x},
$$

where $\tau_{0, x, z}$ are Pauli matrices in band space $(1,2)$ and $\gamma_{x, z}$ in particle-hole space [26]. The bands are assumed to be connected by the hybridization $\xi_{12}$, and $\xi_{ \pm}=\left(\xi_{1} \pm\right.$ $\left.\xi_{2}\right) / 2$, where $\xi_{1(2)}=m_{1(2)} p^{2}-\mu-(-1)^{1(2)} \delta \mu / 2$. The superconducting pairing is spin singlet in each band with the order parameters $\delta_{1(2)}=\delta_{+}+(-) \delta_{-} . F$ in frequency space has been derived in Ref. [25]. We need to transfer them to time representation by contour integration with the choice of the poles as $\mp \varepsilon_{+} \pm i \eta$ and $\mp \varepsilon_{-} \pm i \eta$ with $\eta \rightarrow+0$ (see the detailed derivation for the time representation of $F$ in Appendix B). After Fourier transformation, we obtain for the time difference $t>0$,

$$
\begin{aligned}
& F_{e, \uparrow \downarrow}^{p,-p}(t) \\
& =\frac{i}{\varepsilon_{-}^{2}-\varepsilon_{+}^{2}}\left\{\left(\frac{e^{-i \varepsilon_{+} t}}{\varepsilon_{+}}-\frac{e^{-i \varepsilon_{-} t}}{\varepsilon_{-}}\right)\right. \\
& \quad \times\left[\xi_{12}\left(\delta_{+} \xi_{+}-\delta_{-} \xi_{-}\right) \tau_{x}-\frac{\alpha_{+}}{4}\left(\tau_{0}+\tau_{z}\right)-\frac{\alpha_{-}}{4}\left(\tau_{0}-\tau_{z}\right)\right] \\
& \left.+\frac{1}{2}\left(\delta_{+} \tau_{0}+\delta_{-} \tau_{z}\right)\left(\varepsilon_{+} e^{-i \varepsilon_{+} t}-\varepsilon_{-} e^{-i \varepsilon_{-} t}\right)\right\}, \\
& F_{o, \uparrow \downarrow}^{p,-p}(t)=\frac{\left(e^{-i \varepsilon_{+} t}-e^{-i \varepsilon_{-} t}\right) \delta_{-} \xi_{12}}{\varepsilon_{+}^{2}-\varepsilon_{-}^{2}} \tau_{y},
\end{aligned}
$$

where $\quad \alpha_{ \pm}=\left(\xi_{+} \mp \xi_{-}\right)^{2}\left(\delta_{+} \pm \delta_{-}\right)+\left(\delta_{+} \mp \delta_{-}\right)\left(\xi_{12}^{2}+\right.$ $\left.\delta_{+}^{2}-\delta_{-}^{2}\right) \quad$ and $\quad \varepsilon_{ \pm}^{2}=\xi_{+}^{2}+\xi_{-}^{2}+\xi_{12}^{2}+\delta_{+}^{2}+\delta_{-}^{2} \pm$ $2 \sqrt{\left(\delta_{+} \delta_{-}+\xi_{+} \xi_{-}\right)^{2}+\xi_{12}^{2}\left(\delta_{-}^{2}+\xi_{+}^{2}\right)}$.

From Eq. (11), it follows that the applied pulses must address different electron bands. Otherwise, the signal from the odd-frequency pairing does not contribute to the photoelectron fluctuation signal. This requirement should be feasible if the gap between the bands is much wider than the pulse width. Then, we can adjust $\Omega_{A}=E_{p}+E_{1 \text {,exit }}$, where $E_{1 \text {,exit }}$ constitutes the energy necessary for an electron stemming from band 1 to reach the vacuum energy level, and, likewise, $\Omega_{B}=E_{p}+E_{2, \text { exit }}$ for an electron stemming from band 2 .

Photoelectron fluctuations for two-band model. In Fig. 2, we plot the dependence of the full signal, $\Delta I_{p,-p}^{a b} / \tilde{I}=$ $(1 / 2)\left[\left|F_{\uparrow \downarrow}^{-\boldsymbol{p}, \boldsymbol{p}}\left(t_{B}-t_{A}, 2,1\right)\right|^{2}+\left|F_{\downarrow \uparrow}^{-\boldsymbol{p}, \boldsymbol{p}}\left(t_{B}-t_{A}, 2,1\right)\right|^{2}\right] \quad$ with $\tilde{I}=2\left(S_{0} M_{0}\right)^{4}$ as well as its odd- $\left(\left|F_{o}\right|^{2}\right)$ and even-frequency $\left(\left|F_{e}\right|^{2}\right)$ components with respect to the dimensionless momentum $p / \tilde{p}$ for (a) shorter and (b) longer time differences between the pulses, $t_{B}-t_{A}$. For this model, the following symmetry holds $F_{\sigma, \sigma^{\prime}}=-F_{-\sigma,-\sigma^{\prime}}$. Therefore, the two terms in $\Delta I_{\boldsymbol{p},-\boldsymbol{p}}^{a b}$ are identical. We define the units of energy in such a way that $m_{1} \tilde{p}^{2}=1$. Time differences are shown in units of inverse energy. We use the following parameters: $m_{2} \tilde{p}^{2}=0.5, \delta_{+}=0.03, \delta_{-}=0.02, \xi_{12}=0.05, \mu=$ $0.3, \delta \mu=-0.2$, which correspond to two electronlike bands. In Fig. 2(a), the signal consists of two peaks because the contribution from the even-frequency part is dominating over the odd-frequency one. However, in Fig. 2(b), the signal becomes close to a single peak as the odd-frequency contribution starts to dominate. For $t_{B}-t_{A} \rightarrow 0$, the contribution from the odd-frequency part is vanishing as it should. This analysis demonstrates that the content of the odd- and the even-frequency contributions to superconducting 

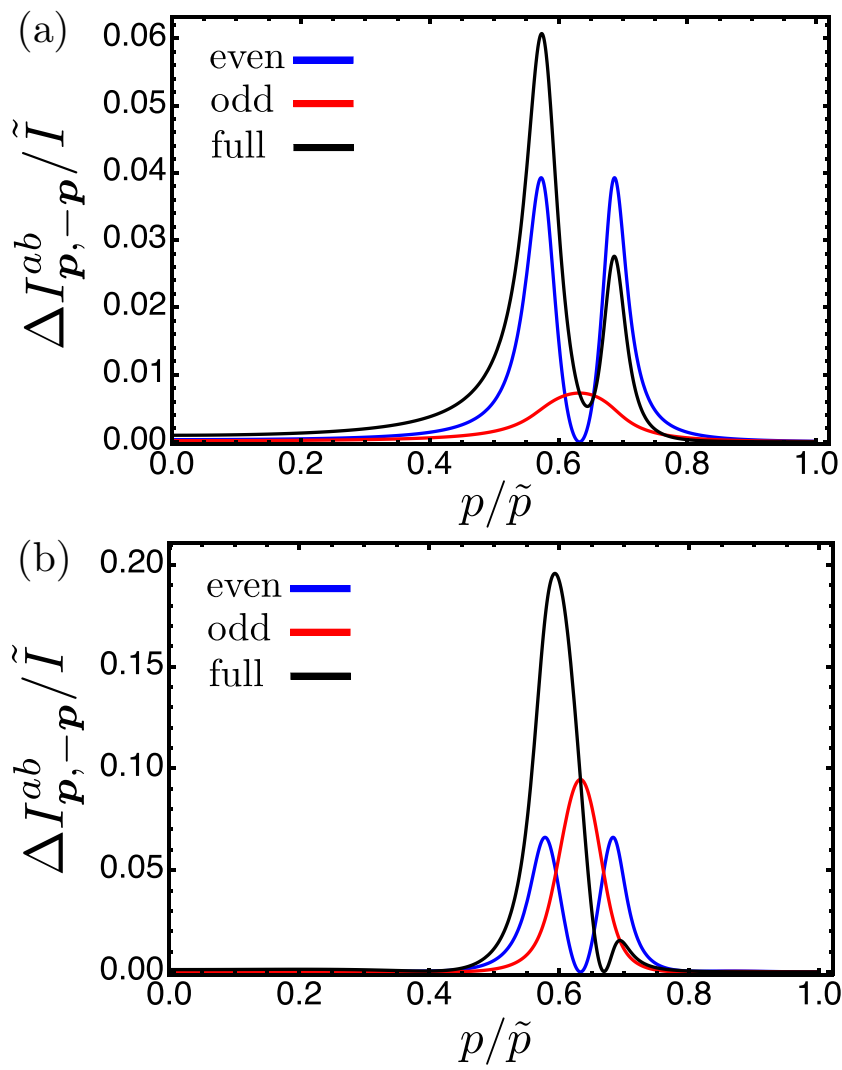

FIG. 2. Momentum dependence of odd (red), even (blue), and full signal (black) for the model of a two-band superconductor for (a) $t_{B}-t_{A}=10$ and (b) $t_{B}-t_{A}=40$. The odd part of the signal has one peak, whereas the even part has two peaks.

pairing can vary as a function of the time argument of the anomalous GF as well as the momenta that appear in it. Note that given momenta fix the corresponding energy. The plots shown in Figs. 2(a) and 2(b) are model-dependent, but we expect that similar features appear in other models as well as real materials.

In Fig. 3, we plot the dependence of the full signal $\Delta I_{\boldsymbol{p},-\boldsymbol{p}}^{a b}$ as well as its even and odd components with respect to the time difference between the pulses $t_{B}-t_{A}$ at a given momentum. Negative time difference $t_{B}-t_{A}$ means that the order of the pulses is exchanged. Thus, we obtain the signal $\Delta I_{\boldsymbol{p},-\boldsymbol{p}}^{a b} / \tilde{I}=\mid F_{o, \uparrow, \downarrow}^{-\boldsymbol{p}, \boldsymbol{p}}\left(t_{B}-t_{A}, 2,1\right)+F_{e, \uparrow, \downarrow}^{-\boldsymbol{p}, \boldsymbol{p}}\left(t_{B}-\right.$ $\left.t_{A}, 2,1\right)\left.\right|^{2}$ for $t_{B}>t_{A}$ and $\Delta I_{p,-p}^{a b} / \tilde{I}=\mid F_{o, \downarrow, \uparrow}^{p,-p}\left(t_{A}-t_{B}, 1,2\right)+$ $\left.F_{e, \downarrow, \uparrow}^{p,-p}\left(t_{A}-t_{B}, 1,2\right)\right|^{2}$ for $t_{A}>t_{B}$. We stress that for the given two-band model the exchange of spin and momentum indices does not change $\Delta I_{\boldsymbol{p},-\boldsymbol{p}}^{a b}$. Only the exchange of band indices provides an additional minus sign due to $F_{o}(1,2)=$ $-F_{o}(2,1)$, yielding different signals for $t_{B}-t_{A}>0$ and $t_{B}-$ $t_{A}<0$.

Note that Fig. 3 illustrates our main result. It shows that the coexistence of even- and odd-frequency pairing results in an asymmetry of the photoelectron fluctuation signal with respect to the exchange of the two pulses. This result is by no means specific to the two-band model under consideration. All it needs for the asymmetry in the photoelectron fluctuation signal to appear is a significant contribution of even- and

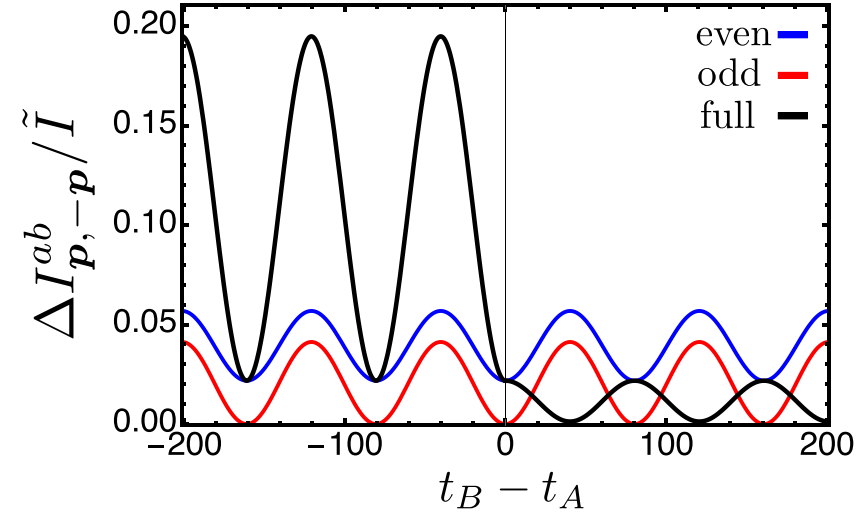

FIG. 3. Time dependence of odd (red), even (blue), and full signal (black) for the model of a two-band superconductor at $p=$ $p_{\max }+\delta p, \delta p / \tilde{p}=0.04$. The full signal is different for a different ordering of pulses $A$ and $B$ since they address different bands. The odd component changes its sign with the order of the band indices, whereas the even one does not.

odd-frequency pairing amplitudes at a certain momentum. A variety of materials fulfills this criterion. Examples thereof are as follows: $\mathrm{MgB}_{2}$ [27], Rashba wires on superconducting substrates [28,29], $\mathrm{Sr}_{2} \mathrm{RuO}_{4}$ [30], iron-pnictide superconductors [31], doped $\mathrm{Bi}_{2} \mathrm{Se}_{3}$ [32,33], $\mathrm{UPt}_{3}$ [15], dilute magnetic superconductors [34], and superconducting Weyl semimetals $[35,36]$.

Experimental issues. Our proposal for detecting timenonlocal pairing via photoelectron fluctuation spectroscopy is based on the following key ingredients: (i) two short probe pulses that both photoemit electrons and do not overlap in time; (ii) probe photon energies that differ by the energetic separation of the bands in a given multiband superconductor [37]; (iii) a detector that measures multihit signals of photoelectrons at opposite momenta. Point (i) is related to the time resolution of state-of-the-art ARPES setups, set by the minimal duration of laser pulses on the order of a few tens of femtoseconds [38]. Point (ii) puts a practical limitation on the energetic separation required in the multiband superconductor. In a probe pulse of short duration, the Heisenberg uncertainty principle imposes a finite energy bandwidth of a photon pulse via $\delta t \delta E \geqslant \hbar \approx 658 \mathrm{meV}$ fs. For a probe duration as short as $30 \mathrm{fs}$, this imposes a requirement that the bands are separated by $20 \mathrm{meV}$ or more at the relevant probe momentum points near the Fermi momentum. We note that this criterion is fulfilled in a variety of multiband superconductors, such as iron pnictides [31] or $\mathrm{Sr}_{2} \mathrm{RuO}_{4}$ [39]. Conditions (i) and (ii) suggest that probe photon energies and probe durations need to be carefully adjusted to the superconductor under consideration. Point (iii) poses a practical challenge to photoelectron detector technologies that are presently available [40]. A similar measurement where a single pulse ejects two photoelectrons (double photoemission) has been demonstrated [41-44]. For our proposal of angle-resolved photoelectron fluctuation spectroscopy, the development of momentum microscopes with multichannel detectors that allow to measure multihit signals appears promising [22].

Summary. In conclusion, we have suggested a measurement scheme based on time- and angle-resolved photoelectron 
fluctuation spectroscopy for the direct detection of oddfrequency superconductivity, a long-standing problem in the field of unconventional superconductivity. We have applied our scheme to a two-band superconductor, analyzed the corresponding signal, and suggested a way to extract the oddfrequency part of it.

Acknowledgments. We acknowledge useful discussions with A. Balatsky, H. Bentmann, T. Heikkilä, K. Nagaev, and L. Rettig. This work was supported by the DFG (Grants No. SPP1666 and No. SFB1170 “ToCoTronics"), the Würzburg-Dresden Cluster of Excellence ct.qmat, EXC2147, Project-ID No. 390858490, and the Elitenetzwerk Bayern Graduate School on "Topological Insulators." M.A.S. acknowledges financial support through the DFG Emmy Noether Program (Grant No. SE 2558/2). F.S. acknowledges support from the Cluster of Excellence "Advanced Imaging of Matter" of the Deutsche Forschungsgemeinschaft (DFG)-EXC 2056-Project-ID No. 390715994.

\section{APPENDIX A: DERIVATION OF THE SIGNAL IN AN EXPERIMENT WITH TWO PROBE PULSES}

The derivation for the case of a single pulse was discussed in Ref. [23]. Here, we describe our derivation for the two nonoverlapping pulses. The Hamiltonian describing interactions between the pulses and the studied material is

$$
H=\sum_{\boldsymbol{k}, \boldsymbol{p}, \sigma, \sigma^{\prime}} S_{A}(t)^{*} e^{i \Omega_{A} t} M_{\boldsymbol{k}, \boldsymbol{p}}^{\sigma, \sigma^{\prime}} a_{\boldsymbol{p} \sigma^{\prime}}^{\dagger} c_{\boldsymbol{k}, \sigma}+S_{B}(t)^{*} e^{i \Omega_{B} t} M_{\boldsymbol{k}, \boldsymbol{p}}^{\sigma, \sigma^{\prime}} b_{\boldsymbol{p} \sigma^{\prime}}^{\dagger} c_{\boldsymbol{k}, \sigma}+\text { H.c. }
$$

The pulse envelope functions $S_{A}(t)$ and $S_{B}(t)$ have finite widths and no overlap. Therefore, we assume that $\left\langle a_{\boldsymbol{p}, \sigma} b_{\boldsymbol{p}, \sigma}^{\dagger}\right\rangle_{0}=$ $\left\langle b_{\boldsymbol{p}, \sigma} a_{\boldsymbol{p}, \sigma}^{\dagger}\right\rangle_{0}=0$. Hence, the vacuum state of emitted electrons can be described as a product of vacuum states for electrons emitted via pulse $A$ and electrons emitted via pulse $B:|0\rangle_{\mathrm{emit}}=|0\rangle_{a}|0\rangle_{b}$.

The total population of the outgoing state is

$$
I_{\boldsymbol{p}, \sigma}^{(1)}=\left\langle n_{\boldsymbol{p}, \sigma}^{a}+n_{\boldsymbol{p}, \sigma}^{b}\right\rangle_{t=\infty} .
$$

The statistical correlation of photoemission events is

$$
I_{\boldsymbol{p}, \sigma ; \boldsymbol{p}^{\prime}, \sigma^{\prime}}^{(2)}=\left\langle\left(n_{\boldsymbol{p}, \sigma}^{a}+n_{\boldsymbol{p}, \sigma}^{b}\right)\left(n_{\boldsymbol{p}^{\prime}, \sigma^{\prime}}^{a}+n_{\boldsymbol{p}^{\prime}, \sigma^{\prime}}^{b}\right)\right\rangle_{t=\infty} .
$$

In terms of the time-evolution matrix, $\mathcal{S}=T \exp \left[-i \int_{-\infty}^{\infty} H(\tau) d \tau\right]$ with $T$ denoting time ordering, we obtain

$$
\begin{aligned}
I_{\boldsymbol{p}, \sigma}^{(1)} & =\left\langle\mathcal{S}^{\dagger}\left(n_{\boldsymbol{p}, \sigma}^{a}+n_{\boldsymbol{p}, \sigma}^{b}\right) \mathcal{S}\right\rangle_{0} \\
& =\left\langle\mathcal{S}_{a}^{\dagger} n_{\boldsymbol{p}, \sigma}^{a} \mathcal{S}_{a}\right\rangle_{0}+\left\langle\mathcal{S}_{b}^{\dagger} n_{\boldsymbol{p}, \sigma}^{b} \mathcal{S}_{b}\right\rangle_{0}, \\
I_{\boldsymbol{p}, \sigma ; \boldsymbol{p}^{\prime}, \sigma^{\prime}}^{(2)} & =\left\langle\mathcal{S}^{\dagger}\left(n_{\boldsymbol{p}, \sigma}^{a}+n_{\boldsymbol{p}, \sigma}^{b}\right)\left(n_{\boldsymbol{p}^{\prime}, \sigma^{\prime}}^{a}+n_{\boldsymbol{p}^{\prime}, \sigma^{\prime}}^{b}\right) \mathcal{S}\right\rangle_{0} \\
& =\left\langle\mathcal{S}^{\dagger}\left(n_{\boldsymbol{p}, \sigma}^{a} n_{\boldsymbol{p}^{\prime}, \sigma^{\prime}}^{a}+n_{\boldsymbol{p}, \sigma}^{a} n_{\boldsymbol{p}^{\prime}, \sigma^{\prime}}^{b}+n_{\boldsymbol{p}, \sigma}^{b} n_{\boldsymbol{p}^{\prime}, \sigma^{\prime}}^{a}+n_{\boldsymbol{p}, \sigma}^{b} n_{\boldsymbol{p}^{\prime}, \sigma^{\prime}}^{b}\right) \mathcal{S}\right\rangle_{0} .
\end{aligned}
$$

The first and the last terms in $I_{p, \sigma ; p^{\prime}, \sigma^{\prime}}^{(2)}$ are the same as in Ref. [23]. Therefore, we will consider only the second and the third terms here. For the case of pulse $B$ being later than pulse $A$, the second term is

$$
\begin{aligned}
I_{\boldsymbol{p}, \sigma ; \boldsymbol{p}^{\prime}, \sigma^{\prime}}^{(2), a b}= & \frac{1}{4}\left\langle\left.\int_{-\infty}^{\infty} d \tau_{1} d \tau_{2} \bar{T}\left[H\left(\tau_{1}\right) H\left(\tau_{2}\right)\right] n_{p \sigma}^{a} n_{p^{\prime} \sigma^{\prime}}^{b} \int_{-\infty}^{\infty} d \tau_{1}^{\prime} d \tau_{2}^{\prime} T\left[H\left(\tau_{1}^{\prime}\right) H\left(\tau_{2}^{\prime}\right)\right]\right|_{0}\right. \\
= & \int_{-\infty}^{\infty} d \tau_{1} d \tau_{2} d \tau_{1}^{\prime} d \tau_{2}^{\prime} \sum_{\substack{k_{1}, p_{1}, \sigma_{1}, \gamma_{1}, k_{1}^{\prime}, p_{1}^{\prime}, \sigma_{1}^{\prime}, \gamma_{1}^{\prime}, k_{2}, p_{2}, \sigma_{2}, \gamma_{2}, k_{2}^{\prime}, p_{2}^{\prime}, \sigma_{2}^{\prime}, \gamma_{2}^{\prime}}} M_{k_{2}, p_{2}}^{\sigma_{2}, \gamma_{2}} M_{k_{1}, p_{1}}^{\sigma_{1}, \gamma_{1}} M_{k_{1}^{\prime}, p_{1}^{\prime}}^{\sigma_{1}^{\prime}, \gamma_{1}^{\prime}} M_{k_{2}^{\prime}, p_{2}^{\prime}}^{\sigma_{2}^{\prime}, \gamma_{2}^{\prime}}\left\langle c_{k_{2}, \sigma_{2}}^{\dagger} c_{k_{1}, \sigma_{1}}^{\dagger} c_{k_{1}^{\prime}, \sigma_{1}^{\prime}} c_{\left.k_{2}^{\prime}, \sigma_{2}^{\prime}\right\rangle_{0}}\right. \\
& \times S_{B}\left(\tau_{1}\right) e^{-i \Omega_{B} \tau_{1}} S_{A}\left(\tau_{2}\right) e^{-i \Omega_{A} \tau_{2}} S_{B}\left(\tau_{1}^{\prime}\right)^{*} e^{i \Omega_{B} \tau_{1}^{\prime}} S_{A}^{*}\left(\tau_{2}^{\prime}\right) e^{i \Omega_{A} \tau_{2}^{\prime}}\left\langle b_{p_{1} \gamma_{1}} b_{p^{\prime} \sigma^{\prime}}^{\dagger}\right\rangle\left\langle b_{p^{\prime} \sigma^{\prime}} b_{p_{1}^{\prime} \gamma_{1}^{\prime}}^{\dagger}\right\rangle\left\langle a_{p_{2} \gamma_{2}} a_{p \sigma}^{\dagger}\right\rangle\left\langle a_{p \sigma} a_{p_{2}^{\prime} \gamma_{2}^{\prime}}^{\dagger}\right\rangle \\
= & \int_{-\infty}^{\infty} d \tau_{1} d \tau_{2} d \tau_{1}^{\prime} d \tau_{2}^{\prime} M_{0}^{4} \\
& \times S_{B}\left(\tau_{1}\right) S_{A}\left(\tau_{2}\right) S_{B}^{*}\left(\tau_{1}^{\prime}\right) S_{A}^{*}\left(\tau_{2}^{\prime}\right) e^{-i \Omega_{B} \tau_{1}-i \Omega_{A} \tau_{2}+i \Omega_{B} \tau_{1}^{\prime}+i \Omega_{A} \tau_{2}^{\prime}+i E_{p^{\prime}}\left(\tau_{1}^{\prime}-\tau_{1}\right)+i E_{p}\left(\tau_{2}^{\prime}-\tau_{2}\right)}\left\langle c_{p, \sigma}^{\dagger}\left(\tau_{2}\right) c_{p^{\prime}, \sigma^{\prime}}^{\dagger}\left(\tau_{1}\right) c_{p^{\prime}, \sigma^{\prime}}\left(\tau_{1}^{\prime}\right) c_{p, \sigma}\left(\tau_{2}^{\prime}\right)\right\rangle_{0}(\mathrm{~A} 6)
\end{aligned}
$$

The symbol $\bar{T}$ denotes antitime ordering due to the fact that we used $\mathcal{S}^{\dagger}$. The last equality sign was taken assuming $M_{\boldsymbol{k}, \boldsymbol{p}}^{\sigma, \sigma^{\prime}}=$ $M_{0} \delta_{k, p} \delta_{\sigma, \sigma^{\prime}}$. Now, we can reintroduce time ordering and obtain a two-point Green's function,

$$
G_{\sigma, \sigma^{\prime}, \sigma^{\prime}, \sigma}^{\boldsymbol{p}, \boldsymbol{p}^{\prime}, \boldsymbol{p}^{\prime}, \boldsymbol{p}}\left(\tau_{2}, \tau_{1}, \tau_{1}^{\prime}, \tau_{2}^{\prime}\right)=\left\langle\bar{T}\left[c_{\boldsymbol{p}, \sigma}^{\dagger}\left(\tau_{2}\right) c_{\boldsymbol{p}^{\prime}, \sigma^{\prime}}^{\dagger}\left(\tau_{1}\right)\right] T\left[c_{\boldsymbol{p}^{\prime}, \sigma^{\prime}}\left(\tau_{1}^{\prime}\right) c_{\boldsymbol{p}, \sigma}\left(\tau_{2}^{\prime}\right)\right]\right\rangle_{0} .
$$




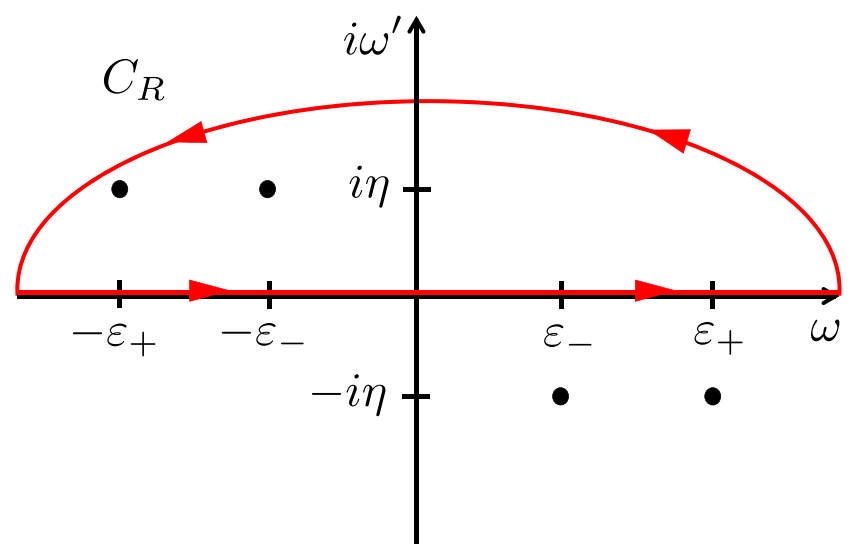

FIG. 4. The contour for integration over frequency.

We can expand it using Wick's theorem,

$$
\begin{aligned}
G_{\sigma, \sigma^{\prime}, \sigma^{\prime}, \sigma}^{\boldsymbol{p}, \boldsymbol{p}^{\prime}, \boldsymbol{p}^{\prime}, \boldsymbol{p}}\left(\tau_{2}, \tau_{1}, \tau_{1}^{\prime}, \tau_{2}^{\prime}\right)= & \left\langle\bar{T}\left[c_{\boldsymbol{p}, \sigma}^{\dagger}\left(\tau_{2}\right) c_{\boldsymbol{p}^{\prime}, \sigma^{\prime}}^{\dagger}\left(\tau_{1}\right)\right] T\left[c_{\boldsymbol{p}^{\prime}, \sigma^{\prime}}\left(\tau_{1}^{\prime}\right) c_{\boldsymbol{p}, \sigma}\left(\tau_{2}^{\prime}\right)\right]\right\rangle_{0} \\
= & \left\langle\bar{T}\left[c_{\boldsymbol{p}, \sigma}^{\dagger}\left(\tau_{2}\right) c_{\boldsymbol{p}^{\prime}, \sigma^{\prime}}^{\dagger}\left(\tau_{1}\right)\right]\right\rangle_{0}\left\langle T\left[c_{\boldsymbol{p}^{\prime}, \sigma^{\prime}}\left(\tau_{1}^{\prime}\right) c_{\boldsymbol{p}, \sigma}\left(\tau_{2}^{\prime}\right)\right]\right\rangle_{0} \\
& -\left\langle c_{\boldsymbol{p}, \sigma}^{\dagger}\left(\tau_{2}\right) c_{\boldsymbol{p}^{\prime}, \sigma^{\prime}}\left(\tau_{1}^{\prime}\right)\right\rangle_{0}\left\langle c_{\boldsymbol{p}^{\prime}, \sigma^{\prime}}^{\dagger}\left(\tau_{1}\right) c_{\boldsymbol{p}, \sigma}\left(\tau_{2}^{\prime}\right)\right\rangle_{0} \\
& +\left\langle c_{\boldsymbol{p}, \sigma}^{\dagger}\left(\tau_{2}\right) c_{\boldsymbol{p}, \sigma}\left(\tau_{2}^{\prime}\right)\right\rangle_{0}\left\langle c_{\boldsymbol{p}^{\prime}, \sigma^{\prime}}^{\dagger}\left(\tau_{1}\right) c_{\boldsymbol{p}^{\prime}, \sigma^{\prime}}\left(\tau_{1}^{\prime}\right)\right\rangle_{0} .
\end{aligned}
$$

If the Hamiltonian describing our material does not contain terms proportional to $c_{\boldsymbol{p}, \sigma}^{\dagger} c_{\boldsymbol{p}^{\prime}, \sigma^{\prime}}$ for $\boldsymbol{p} \neq \boldsymbol{p}^{\prime}$ and $\sigma \neq \sigma^{\prime}$, which is often the case for a clean material, the second term is zero in the case of $\boldsymbol{p}^{\prime}=-\boldsymbol{p}$. The last term is a product of two one-point Green's functions.

Thus, for the fluctuations $\Delta I_{\boldsymbol{p}, \sigma ;-\boldsymbol{p}, \sigma^{\prime}}^{a b}=I_{\boldsymbol{p}, \sigma ;-\boldsymbol{p}, \sigma^{\prime}}^{(2), a b}-I_{\boldsymbol{p}, \sigma}^{(1), a} I_{-\boldsymbol{p}, \sigma^{\prime}}^{(1), b}=\left\langle n_{\boldsymbol{p}, \sigma}^{a} n_{-\boldsymbol{p}, \sigma^{\prime}}^{b}\right\rangle_{t=\infty}-\left\langle n_{\boldsymbol{p}, \sigma}^{a}\right\rangle_{t=\infty}\left\langle n_{-\boldsymbol{p}, \sigma^{\prime}}^{b}\right\rangle_{t=\infty}$, we obtain

$$
\Delta I_{\boldsymbol{p}, \sigma ;-\boldsymbol{p}, \sigma^{\prime}}^{a b}=\left|\int_{-\infty}^{\infty} d \tau_{1} d \tau_{2} M_{0}^{2} S_{B}^{*}\left(\tau_{1}\right) S_{A}^{*}\left(\tau_{2}\right) e^{i \Omega_{B} \tau_{1}+i \Omega_{A} \tau_{2}+i E_{p}\left(\tau_{1}+\tau_{2}\right)}\left\langle T c_{-\boldsymbol{p}, \sigma^{\prime}}\left(\tau_{1}\right) c_{\boldsymbol{p}, \sigma}\left(\tau_{2}\right)\right\rangle_{0}\right|^{2} .
$$

In the case of a single pulse, the signal has the same form (with all indices $A$ and $B$ being absent) [23], even though the derivation is slightly different due to the fact that all emitted electrons have the same vacuum state. In Eqs. (6) and (7) and the related discussion in the main text, we present the above equation.

In case of $\delta$-function-shaped envelopes of pulses, $S_{A}(t)=S_{0} \delta\left(t-t_{A}\right), S_{B}(t)=S_{0} \delta\left(t-t_{B}\right)$, the signal is simplified into

$$
\Delta I_{\boldsymbol{p}, \sigma ;-\boldsymbol{p}, \sigma^{\prime}}^{a b}=M_{0}^{4} S_{0}^{4}\left\langle c_{-\boldsymbol{p}, \sigma^{\prime}}\left(t_{B}\right) c_{\boldsymbol{p}, \sigma}\left(t_{A}\right)\right\rangle_{0}=M_{0}^{4} S_{0}^{4}\left|F_{\sigma^{\prime}, \sigma}^{-\boldsymbol{p}, \boldsymbol{p}}\left(t_{B}, t_{A}\right)\right|^{2} .
$$

This is Eq. (8) from the main text. If operators $c$ have an additional index, e.g., a band index, the anomalous Green's function also acquires indices. In the main text, we consider an example of a two-band superconductor when pulse $A$ affects band 1 and pulse $B$ affects band 2 . Therefore, the signal is

$$
\Delta I_{\boldsymbol{p}, \sigma ;-\boldsymbol{p}, \sigma^{\prime}}^{a b}=M_{0}^{4} S_{0}^{4}\left\langle c_{2,-\boldsymbol{p}, \sigma^{\prime}}\left(t_{B}\right) c_{1, \boldsymbol{p}, \sigma}\left(t_{A}\right)\right\rangle_{0}=M_{0}^{4} S_{0}^{4}\left|F_{\sigma^{\prime}, \sigma}^{-\boldsymbol{p}, \boldsymbol{p}}\left(t_{B}, t_{A}, 2,1\right)\right|^{2} .
$$

The fact that the pulses address different bands provides interesting results for the dependence of the signal on the time difference between pulses, see Fig. 3 of the main text.

\section{APPENDIX B: TIME DEPENDENCE OF ANOMALOUS GREEN'S FUNCTIONS OF A TWO-BAND SUPERCONDUCTOR}

In this Appendix, we present the way we perform the Fourier transformation of the anomalous Green's functions, even and odd, for a model of a two-band superconductor described in Ref. [25].

To obtain anomalous Green's functions in time representation, we need to calculate $\int_{-\infty}^{\infty} F(\omega) e^{i \omega t} d \omega$, however, this integral has divergencies at $\pm \varepsilon_{ \pm}$, see Eqs. (5) and (6) in Ref. [25]. Therefore, we employ the circling of the poles analogously to the Landau theorem $[45,46]$. As the Landau theorem applies to a single type of fermions, whereas we have two bands and, thus, a combination of their contributions into the Green's function, we use it for the denominators respective to different eigenbands,

$$
D=\left(\omega^{2}-\varepsilon_{-}^{2}\right)\left(\omega^{2}-\varepsilon_{+}^{2}\right)=\left(\omega-\varepsilon_{-}+i \eta\right)\left(\omega+\varepsilon_{-}-i \eta\right)\left(\omega-\varepsilon_{+}+i \eta\right)\left(\omega+\varepsilon_{+}-i \eta\right) .
$$

Thus, the poles are at $\omega_{1,2}=\mp \varepsilon_{+} \pm i \eta$ and $\omega_{3,4}=\mp \varepsilon_{-} \pm i \eta$. Then, we define the contour in the complex plane $\Omega=\left\{\omega, i \omega^{\prime}\right\}$, see Fig. 4. 
The contour has radius $R$ and is defined for the upper half plane. We obtain

$$
\int_{C} F(\Omega) e^{i \Omega t} d \Omega=\int_{-R}^{R} F(\Omega) e^{i \Omega t} d \Omega+\int_{C_{R}} F(\Omega) e^{i \Omega t} d \Omega=2 \pi i \sum_{\omega_{1}, \omega_{3}} \operatorname{res}\left[F e^{i \Omega t}\right] .
$$

Let us evaluate the second integral (see, e.g., Ref. [47]). First of all, for large enough $|\Omega|>R_{0}$,

$$
F(\Omega)=\Omega^{-2}[1+O(1 / \Omega)] .
$$

We can also find such $R_{1} \geqslant R_{0}$ that at all $|\Omega| \geqslant R_{1}$ we have $|1+O(1 / \Omega)| \leqslant 2$, i.e., $|F(\Omega)| \leqslant 2|\Omega|^{-2}$. Moreover,

$$
\left|e^{i \Omega t}\right|=\left|e^{i\left(\omega+i \omega^{\prime}\right) t}\right|=e^{-\omega^{\prime} t}=e^{-t R \sin \phi} .
$$

This equality holds for $t>0$. Note that $\sin \phi \geqslant \frac{2}{\pi} \phi$ for $\phi \in[0, \pi / 2]$. Thus, for $R \geqslant R_{1}$ we have

$$
\begin{aligned}
\left|\int_{C_{R}} F(\Omega) e^{i \Omega t} d \Omega\right| & \leqslant \int_{C_{R}} 2|\Omega|^{-2} e^{-t R \sin \phi}|d \Omega| \\
& \leqslant 2 R^{-2} R \int_{0}^{\pi} e^{-\tau R \sin \phi} d \phi \\
& \leqslant 2 R^{-2} R 2 \int_{0}^{\pi / 2} e^{-2 \tau R \phi / \pi} d \phi \leqslant 2 R^{-2} \frac{\pi}{\tau} \rightarrow_{R \rightarrow \infty} 0 .
\end{aligned}
$$

In such a way we obtain

$$
\int_{-\infty}^{\infty} F(\Omega) e^{i \Omega t} d \Omega=2 \pi i \sum_{\omega_{1}, \omega_{3}} \operatorname{res}\left[F e^{i \Omega t}\right]
$$

Next we calculate the residues. As these are simply poles of the first order, we obtain for the even component of the signal $F_{e}$,

$$
\begin{aligned}
\operatorname{res}_{\omega_{1}}\left[F_{e}(\Omega) e^{i \Omega t}\right] & =\frac{e^{i \omega_{1} t}}{\left(\omega_{1}-\varepsilon_{+}\right)\left(\omega_{1}^{2}-\varepsilon_{-}^{2}\right)}\left(\begin{array}{cc}
\left(\delta_{+}+\delta_{-}\right) \omega_{1}^{2}-\alpha_{+} & 2 \xi_{12}\left(\delta_{+} \xi_{+}-\delta_{-} \xi_{-}\right) \\
2 \xi_{12}\left(\delta_{+} \xi_{+}-\delta_{-} \xi_{-}\right) & \left(\delta_{+}-\delta_{-}\right) \omega_{1}^{2}-\alpha_{-}
\end{array}\right), \\
\operatorname{res}_{\omega_{3}}\left[F_{e}(\Omega) e^{i \Omega t}\right] & =\frac{e^{i \omega_{3} t}}{\left(\omega_{3}-\varepsilon_{-}\right)\left(\omega_{3}^{2}-\varepsilon_{+}^{2}\right)}\left(\begin{array}{cc}
\left(\delta_{+}+\delta_{-}\right) \omega_{3}^{2}-\alpha_{+} & 2 \xi_{12}\left(\delta_{+} \xi_{+}-\delta_{-} \xi_{-}\right) \\
2 \xi_{12}\left(\delta_{+} \xi_{+}-\delta_{-} \xi_{-}\right) & \left(\delta_{+}-\delta_{-}\right) \omega_{3}^{2}-\alpha_{-}
\end{array}\right) .
\end{aligned}
$$

Then, we take a limit $\eta \rightarrow 0$ and obtain

$$
\begin{aligned}
F_{e}(t)= & \frac{1}{2 \pi} \int_{-\infty}^{\infty} F_{e}(\Omega) e^{i \Omega t} d \Omega \\
= & \frac{i}{2\left(\varepsilon_{-}^{2}-\varepsilon_{+}^{2}\right)}\left[\frac{e^{-i \varepsilon_{+} t}}{\varepsilon_{+}}\left(\begin{array}{cc}
\left(\delta_{+}+\delta_{-}\right) \varepsilon_{+}^{2}-\alpha_{+} & 2 \xi_{12}\left(\delta_{+} \xi_{+}-\delta_{-} \xi_{-}\right) \\
2 \xi_{12}\left(\delta_{+} \xi_{+}-\delta_{-} \xi_{-}\right) & \left(\delta_{+}-\delta_{-}\right) \varepsilon_{+}^{2}-\alpha_{-}
\end{array}\right)\right. \\
& \left.-\frac{e^{-i \varepsilon_{-} t}}{\varepsilon_{-}}\left(\begin{array}{cc}
\left(\delta_{+}+\delta_{-}\right) \varepsilon_{-}^{2}-\alpha_{+} & 2 \xi_{12}\left(\delta_{+} \xi_{+}-\delta_{-} \xi_{-}\right) \\
2 \xi_{12}\left(\delta_{+} \xi_{+}-\delta_{-} \xi_{-}\right) & \left(\delta_{+}-\delta_{-}\right) \varepsilon_{-}^{2}-\alpha_{-}
\end{array}\right)\right] .
\end{aligned}
$$

Analogously, for the odd component $F_{o}(t)$, we obtain

$$
F_{o}(t)=\frac{1}{2 \pi} \int_{-\infty}^{\infty} F_{o}(\Omega) e^{i \Omega t} d \Omega=\frac{i\left(e^{-i \varepsilon_{+} t}-e^{-i \varepsilon_{-} t}\right)}{\varepsilon_{+}^{2}-\varepsilon_{-}^{2}}\left(\begin{array}{cc}
0 & -\delta_{-} \xi_{12} \\
\delta_{-} \xi_{12} & 0
\end{array}\right) .
$$

Note that Eqs. (B9) and (B10) correspond to Eqs. (10) and (11) of the main text. We simplify the indices of the anomalous Green's functions here for ease of notation, keeping only time and even/odd notation.

[1] V. Berezinskii, Novaya model' anizotropnoy fazy sverhtekuchego $\mathrm{He}_{3}$, Pis'ma Zh. Eksp. Teor. Fiz. 20, 628 (1974).

[2] A. Balatsky and E. Abrahams, New class of singlet superconductors which break the time reversal and parity, Phys. Rev. B 45, 13125 (1992).

[3] T. R. Kirkpatrick and D. Belitz, Disorder-Induced Triplet Superconductivity, Phys. Rev. Lett. 66, 1533 (1991).
[4] V. J. Emery and S. Kivelson, Mapping of the two-channel Kondo problem to a resonant-level model, Phys. Rev. B 46, 10812 (1992).

[5] P. Coleman, E. Miranda, and A. Tsvelik, Possible Realization of Odd-Frequency Pairing in Heavy Fermion Compounds, Phys. Rev. Lett. 70, 2960 (1993). 
[6] P. Coleman, A. Georges, and, A. M. Tsvelik, Reflections on the one-dimensional realization of odd-frequency pairing, J. Phys.: Condens. Matter 9, 345 (1997).

[7] Y. Fuseya, H. Kohno, and K. Miyake, Realization of OddFrequency p-Wave SpinâSinglet Superconductivity Coexisting with Antiferromagnetic Order near Quantum Critical Point, J. Phys. Soc. Jpn. 72, 2914 (2003).

[8] F. S. Bergeret, A. F. Volkov, and K. B. Efetov, Odd triplet superconductivity and related phenomena in superconductorferromagnet structures, Rev. Mod. Phys. 77, 1321 (2005).

[9] Y. Tanaka, M. Sato, and N. Nagaosa, Symmetry and topology in superconductors odd-frequency pairing and edge states, J. Phys. Soc. Jpn 81, 011013 (2012).

[10] J. Linder and A. V. Balatsky, Odd-frequency superconductivity, Rev. Mod. Phys. 91, 045005 (2019).

[11] J. Cayao, C. Triola, and A. M. Black-Schaffer, Odd-frequency superconducting pairing in one-dimensional systems, Eur. Phys. J.: Spec. Top. 229, 545 (2020).

[12] T. S. Khaire, M. A. Khasawneh, W. P. Pratt, Jr., and N. O. Birge, Observation of Spin-Triplet Superconductivity in Co-Based Josephson Junctions, Phys. Rev. Lett. 104, 137002 (2010).

[13] E. R. Schemm, W. J. Gannon, C. M. Wishne, W. P. Halperin, and A. Kapitulnik, Observation of broken time-reversal symmetry in the heavy-fermion superconductor $\mathrm{UPt}_{3}$, Science 345, 190 (2014).

[14] Z. Wang, J. Berlinsky, G. Zwicknagl, and C. Kallin, Intrinsic ac anomalous Hall effect of nonsymmorphic chiral superconductors with an application to $\mathrm{UPt}_{3}$, Phys. Rev. B 96, 174511 (2017).

[15] C. Triola and A. M. Black-Schaffer, Odd-frequency pairing and Kerr effect in the heavy-fermion superconductor $\mathrm{UPt}_{3}$, Phys. Rev. B 97, 064505 (2018).

[16] A. Di Bernardo, Z. Salman, X. L. Wang, M. Amado, M. Egilmez, M. G. Flokstra, A. Suter, S. L. Lee, J. H. Zhao, T. Prokscha, E. Morenzoni, M. G. Blamire, J. Linder, and J. W. A. Robinson, Intrinsic Paramagnetic Meissner Effect Due to s-Wave Odd-Frequency Superconductivity, Phys. Rev. X 5, 041021 (2015).

[17] V. Perrin, F. L. N. Santos, G. C. Menard, C. Brun, T. Cren, M. Civelli, and P. Simon, Unveiling Odd-Frequency Pairing around a Magnetic Impurity in a Superconductor, Phys. Rev. Lett. 125, 117003 (2020).

[18] O. Kashuba, B. Sothmann, P. Burset, and B. Trauzettel, Majorana STM as a perfect detector of odd-frequency superconductivity, Phys. Rev. B 95, 174516 (2017).

[19] C. Fleckenstein, N. Traverso Ziani, and B. Trauzettel, Conductance signatures of odd-frequency superconductivity in quantum spin Hall systems using a quantum point contact, Phys. Rev. B 97, 134523 (2018).

[20] P. Dutta and A. M. Black-Schaffer, Signature of odd-frequency equal-spin triplet pairing in the Josephson current on the surface of Weyl nodal loop semimetals, Phys. Rev. B 100, 104511 (2019).

[21] R. Seoane Souto, D. Kuzmanovski, and A. V. Balatsky, Signatures of odd-frequency pairing in the Josephson junction current noise, Phys. Rev. Res. 2, 043193 (2020).

[22] D. Kutnyakhov, R. P. Xian, M. Dendzik, M. Heber, F. Pressacco, S. Y. Agustsson, L. Wenthaus, H. Meyer, S. Gieschen, G. Mercurio, A. Benz, K. Bühlman, S. Däster, R.
Gort, D. Curcio, K. Volckaert, M. Bianchi, Ch. Sanders, J. A. Miwa, S. Ulstrup, A. Oelsner et al., Time- and momentumresolved photoemission studies using time-of-flight momentum microscopy at a free-electron laser, Rev. Sci. Instrum. 91, 013109 (2020).

[23] C. Stahl and M. Eckstein, Noise correlations in time- and angle-resolved photoemission spectroscopy, Phys. Rev. B 99, 241111(R) (2019).

[24] This assumption reflects an experiment in which the temporal line width of the pulse is the shortest timescale of the problem. It should be kept in mind that a small linewidth might cause problems in the detection scheme if too many energy levels are excited due to energy-time uncertainty. In this case, the linewidth should be adapted to the problem. However, the principle of our detection scheme does not rely on this assumption.

[25] F. Parhizgar and A. M. Black-Schaffer, Diamagnetic and paramagnetic Meissner effect from odd-frequency pairing in multiorbital superconductors, Phys. Rev. B 104, 054507 (2021).

[26] Note that the operators $c$ that appear, for instance, in the Hamiltonian (1) need to be supplemented by an additional band index if we deal with a multiband superconductor.

[27] A. Aperis, P. Maldonado, and P. M. Oppeneer, Ab initio theory of magnetic-field-induced odd-frequency two-band superconductivity in $\mathrm{MgB}_{2}$, Phys. Rev. B 92, 054516 (2015).

[28] H. Ebisu, K. Yada, H. Kasai, and Y. Tanaka, Odd-frequency pairing in topological superconductivity in a one-dimensional magnetic chain, Phys. Rev. B 91, 054518 (2015).

[29] D. Takagi, S. Tamura, and Y. Tanaka, Odd-frequency pairing and proximity effect in Kitaev chain systems including a topological critical point, Phys. Rev. B 101, 024509 (2020).

[30] L. Komendová and A. M. Black-Schaffer, Odd-Frequency Superconductivity in $\mathrm{Sr}_{2} \mathrm{RuO}_{4}$ Measured by Kerr Rotation, Phys. Rev. Lett. 119, 087001 (2017).

[31] E. M. Nica, R. Yu, and Q. Si, Orbital-selective pairing and superconductivity in iron selenides, npj Quantum Mater. 2, 24 (2017).

[32] F. Parhizgar and A. M. Black-Schaffer, Highly tunable timereversal-invariant topological superconductivity in topological insulator thin films, Sci. Rep. 7, 9817 (2017).

[33] J. Schmidt, F. Parhizgar, and A. M. Black-Schaffer, Oddfrequency superconductivity and Meissner effect in the doped topological insulator $\mathrm{Bi}_{2} \mathrm{Se}_{3}$, Phys. Rev. B 101, 180512(R) (2020).

[34] F. L. N. Santos, V. Perrin, F. Jamet, M. Civelli, P. Simon, M. C. O. Aguiar, E. Miranda, and M. J. Rozenberg, Oddfrequency superconductivity in dilute magnetic superconductors, Phys. Rev. Res. 2, 033229 (2020).

[35] F. Parhizgar and A. M. Black-Schaffer, Large Josephson current in Weyl nodal loop semimetals due to odd-frequency superconductivity, npj Quantum Mater. 5, 42 (2020).

[36] P. Dutta, F. Parhizgar, and A. M. Black-Schaffer, Finite bulk Josephson currents and chirality blockade removal from interorbital pairing in magnetic Weyl semimetals, Phys. Rev. B 101, 064514 (2020).

[37] This criterion only applies to the multiband case. In the singleband case, other ways to distinguish the two probe pulses than energy resolution have to be employed. This could, for instance, be polarized probe pulses in combination with selection rules. 
[38] A. de la Torre, D. M. Kennes, M. Claassen, S. Gerber, J. W. McIver, and M. A. Sentef, Nonthermal pathways to ultrafast control in quantum materials, Rev. Mod. Phys. 93, 041002 (2021).

[39] A. Tamai, M. Zingl, E. Rozbicki, E. Cappelli, S. Ricco, A. de la Torre, S. McKeown Walker, F. Y. Bruno, P. D. C. King, W. Meevasana, M. Shi, M. Radovic, N. C. Plumb, A. S. Gibbs, A. P. Mackenzie, C. Berthod, H. U. R. Strand, M. Kim, A. Georges, and F. Baumberger, High-Resolution Photoemission on $\mathrm{Sr}_{2} \mathrm{RuO}_{4}$ Reveals Correlation-Enhanced Effective SpinOrbit Coupling and Dominantly Local Self-Energies, Phys. Rev. X 9, 021048 (2019).

[40] J. A. Sobota, Y. He, and Z.-X. Shen, Angle-resolved photoemission studies of quantum materials, Rev. Mod. Phys. 93, 025006 (2021).

[41] F. O. Schumann, C. Winkler, and J. Kirschner, Correlation Effects in Two Electron Photoemission, Phys. Rev. Lett. 98, 257604 (2007).
[42] F. O. Schumann, C. Winkler, G. Kerherve, and J. Kirschner, Mapping the electron correlation in two-electron photoemission, Phys. Rev. B 73, 041404(R) (2006).

[43] A. Trützschler, M. Huth, C.-T. Chiang, R. Kamrla, F. O Schumann, J. Kirschner, and W. Widdra, Band-Resolved Double Photoemission Spectroscopy on Correlated Valence Electron Pairs in Metals, Phys. Rev. Lett. 118, 136401 (2017).

[44] C.-T. Chiang, A. Trützschler, M. Huth, R. Kamrla, F. O. Schumann, W. Widdra, Laser-based double photoemission spectroscopy at surfaces, Prog. Surf. Sci. 95, 100572 (2020).

[45] L. D. Landau, The properties of the Green function for particles in staistics, Sov. JETP 7, 182 (1958).

[46] L. P. Gor'kov, On the energy spectrum of superconductors, Sov. JETP 7, 505 (1958).

[47] E. S. Polovinkin, Lecture Course on the Theory of Functions of a Complex Variable (Moscow Institute of Physics and Technology, Moscow, 1999). 